\title{
A Review on Low Light Video Enhancement Using Image Processing Technique
}

\author{
Ms. Pallavi H. Yawalkar ${ }^{1}$, Mr. P. N. Pusdekar ${ }^{2}$ \\ Student, Dept. of Electronics and Telecommunication, P.R Pote (Patil) College of Engineering and Management, \\ Amravati, India ${ }^{1}$ \\ Professor, Dept. of Electronics and Telecommunication, P.R Pote (Patil) College of Engineering and Management, \\ Amravati, India ${ }^{2}$
}

\begin{abstract}
Over the several decades, there have been notable capability improvements in Digital cameras including resolution and sensitivity. Despite these improvements, however, modern digital cameras are still limited in capturing high dynamic range images in low-light conditions. These cameras often rely on automatic exposure control to capture image of high dynamic range, but the longer exposure time often results motion blur. Many approaches are developed for enhancing low light video; however most of them consider video from moderately dark conditions. In this project, we propose an effective framework approach to enhance video from low light environments using appropriate noise removal filter technique which will maintain image quality.
\end{abstract}

Keywords: Video, processing, enhancement, Image, MATLAB.

\section{INTRODUCTION}

Digital video has become an integral part of everyday life. It is well-known that video enhancement as an active topic in computer vision has received much attention in recent years. The aim is to improve the visual appearance of the video, or to provide a "better" transform representation for future automated video processing, such as analysis, detection, segmentation, and recognition. Moreover, it helps analyses background information that is essential to understand object behaviour without requiring expensive human visual inspection. There are numerous applications where digital video is acquired, processed and used, such as surveillance, general identity verification, criminal justice systems, civilian or military video processing.

More and more video cameras are widely deployed in many scenarios e.g. Public places, production Plants, domestic surveillance systems etc. Most of the video cameras work in the open air which means the quality of video depends on the weather conditions. The camera and video surveillance systems are expected effective in all lighting and weather conditions, but the majority of these cameras were not designed for low-lighting, therefore the poor capture quality of video camera makes the video unusable for many applications in bad conditions e.g. dark night, soaking rain, heavy snow and fog. Over the last several decades, there have been substantial capability improvements in digital cameras including resolutions and sensitivity. Despite these improvements, however, modern digital cameras are still limited in capturing high dynamic range images in low-light conditions. These cameras often rely on automatic exposure control to capture images of high dynamic range, but the longer exposure time often results motion blur. Additionally, image sequences captured in low-light conditions often have low signal-tonoise ratio (SNR). When the illumination is very low, the level of noise becomes relatively higher than the signal, so conventional denoising techniques cannot be applied.
To design an effective and fast low lighting video enhancement is a challenging problem. Many approaches are developed for enhancing low-light video however most of them consider video from moderately dark conditions. In this project, we aim to develop a novel framework to enhance video from extremely low-light environments. Our method consists of temporal noise reduction, contrast enhancement and denoising. The Software tool used is MATLAB.

\section{LITERATURE REVIEW}

Henrik Malm Magnus Oskarsson Eric Warrant [1] presented a methodology for adaptive enhancement and noise reduction for very dark image sequences with very low dynamic range. The approach is very general and adapts to the spatiotemporal intensity structure in order to prevent motion blur and smoothing across important structural edges. The method also includes a sharpening feature which prevents the most important object contours from being over-smoothed. Most parameters can be set generally for a very large group of input sequences. These parameters include: the clip-limit in the contrast-limited histogram equalization, the maximum and minimum widths of the filtering kernels and the width of the isotropic smoothing of the structure tensor and in the gradient calculations. However, the scaling parameter for the width function has to be adjusted to the noise level in the current sequence. The best approach when applying the method to colour images has been discussed, which includes demosaicing from the Bayer pattern in raw input colour data simultaneously to the noise reduction. They implemented the method using a GPU and achieved interactive performance.

Qing Xu1, 2, Hailin Jiang1, Riccardo Scopigno3, and Mateu Sbert4 [2] presented a novel three-stage algorithm for very low-light video denoising and enhancement. A 
new framework for very dark videos denoising and enhancement has been introduced and shown to largely improve current state-of-the-art results.

Jinhui $\mathrm{Hu}$, Ruimin $\mathrm{Hu}$, Zhongyuan Wang, Yan Gong, MangDuan [3] presented a technique of kinect depth based method for low light surveillance image enhancement. Pre-processing for Kinect depth map, depth constrained non-local means denoising and depth aware contrast stretching are performed successively in this algorithm to promote the visual quality for low light surveillance image. Comparing with the previous works, this method is able to enlarge the low dynamic range and promote both globe and local depth perception for the low light surveillance image meanwhile. The experimental results show that this method generates clearer object edges and more distinct depth perception for enhanced lowlight surveillance images. High noise level from darkness and low dynamic range are two characteristics of low light surveillance image that severely degrade the visual quality.

Minjae Kim1, Student Member, IEEE, Dubok Park1, David K. Han2, and Hanseok Ko1 [4] proposed a novel framework for enhancement of very low-light video. For noise reduction, motion adaptive temporal filtering based on the Kalman structured updating is presented. Dynamic range of denoised video is increased by adaptive adjustment of RGB histograms. Finally, remaining noise is removed using Non-local means (NLM) denoising. The proposed method exploits color filter array (CFA) raw data for achieving low memory consumption. Histogram adjustment using the gamma transform and the adaptive clipping threshold is also presented to increase the dynamic range of the low-light video. The experimental results indicate that this method is highly promising for real time applications to consumer digital cameras, especially CCTV and the surveillance video system.

\section{III.PROPOSED WORK}

The video enhancement is still an active area of research by many experts. There are still many problems of video enhancement, such as false background problem, colour shift problem etc. Video enhancement is one of the most important and difficult component of video security surveillance system. The increasing use of night operations requires more details and integrated information from the enhanced image. However, low quality video of most surveillance cameras is not satisfied and difficult to understand because they lack surrounding scene context due to poor illumination. So one of key problems is image/frame fusion problem to ensure better image reconstruction and colour assignment. A large number of techniques have been proposed to address this problem.

In this we focus on the existing techniques of video enhancement, which can be made better in poor visibility light

condition. Desired outcome of the project is to enhance video. The steps to get desired outcome is as shown in fig.1.
Low light video

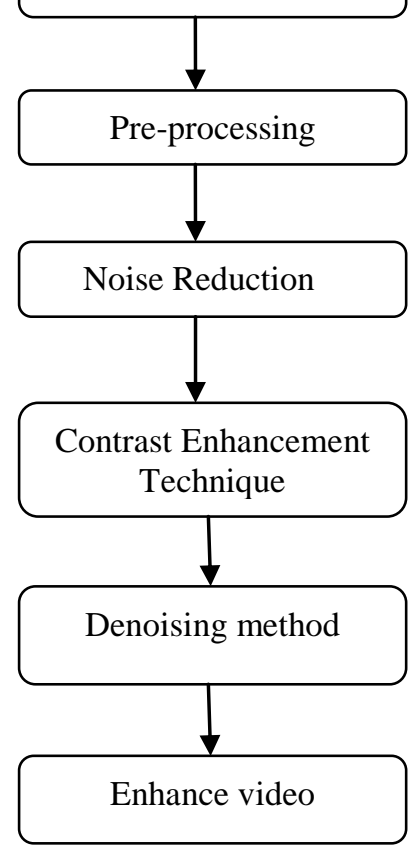

Fig. 1. Flow to get enhance video

\section{A. Pre-processing}

The low light video is applied to the first step which is pre-processing. Pre-processing is the term for operations on images at the lowest level of abstraction. These operations do not increase image information content but they decrease it if entropy is an information measure. The aim of pre-processing is an improvement of the image data that suppresses undesired distortions or enhances some image features in video relevant for further processing and analysis task. Image pre-processing use the redundancy in images. The low light video is applied to the first step which is pre-processing.

\section{B. Noise reduction}

Noise is the result of errors in the image acquisition process that result in pixel values that do not reflect the true intensities of the real scene.

Since image sequences are temporally correlated, noise can be reduced effectively by temporal filtering. Videos are prone to a variety of types of noise. The output from first stage is given to the noise reduction. Noise reduction is the process of removing noise from a signal.

\section{Contrast enhancement technique}

The contrast of an image is a measure of its dynamic range, or the "spread" of its histogram. The dynamic range of an image is defined to be the entire range of intensity values contained within an image, or put a simpler way, the maximum pixel value minus the minimum pixel value. Contrast enhancements improve the perceptibility of objects in the scene by enhancing the brightness difference between objects and their backgrounds. Contrast enhancements are typically performed as a contrast stretch 
followed by a tonal enhancement, although these could both be performed in one step. Image enhancement is the process of adjusting images so that the results are more suitable for display or further image analysis.

A contrast stretch improves the brightness differences uniformly across the dynamic range of the image, whereas tonal enhancements improve the brightness differences in the shadow (dark), midtone (grays), or highlight (bright) regions at the expense of the brightness differences in the other regions. After the noise reduction, we have to amplify the intensity of the low light video. This stage is used to enhance the contrast of low light video. It is also called as tone mapping. Contrast enhancement processes adjust the relative brightness and darkness of objects in the scene to improve their visibility.

\section{Denoising method}

For the final step of low light video enhancement we have to apply filtering for smoothing the remaining noise. Even though most of the noise is removed by the noise reduction, the noise is introduced by tone mapping step. Moreover, since the level of the noise is much higher than the low light environment, edges and textures are often over smoothed during the denoising process.

\section{IV.CONCLUSION}

The MATLAB brings to digital image processing is an extensive set of functions for processing multidimensional arrays of which images (two-dimensional numerical arrays) are a special case. The Image Processing Toolbox is a collection of functions that extend the capability of the MATLAB numeric computing environment. These functions, and the expressiveness of the MATLAB language, make video-processing operations easy to write in a compact, clear manner, thus providing an ideal software prototyping environment for the solution of video processing problems. The previously used methodologies are able to reduce the noise as well as increase the contrast level of the video but used methods are not still effectively work on colour video. Therefore our aim to get clear video from the low light video.

\section{ACKNOWLEDGMENT}

I would like to take this opportunity to express my profound gratitude and deep regard to Prof.P.N.Pusdekar, for his exemplary guidance, valuable feedback and constant encouragement throughout the duration of the proposed work. His valuable suggestions were of immense help throughout my work. His perceptive criticism kept me working to make this project in a much better way. Working under him was an extremely knowledgeable experience for me. And I also thanks to my family members for supporting me.

\section{REFERENCES}

[1] Henrik Malm Magnus Oskarsson Eric Warrant "Adaptive enhancement and noise reduction in very low light-level video"9781-4244-1631-8/07/\$25.00 @2007 IEEE.

[2] Qing Xu1, 2, Hailin Jiang1, Riccardo Scopigno3, and Mateu Sbert4 "A New Approach ForVery Dark Video Denoising And
Enhancement"Proceedings of $2010 \quad$ IEEE $17^{\text {th }}$ International Conference on Image Processing September 26-29, 2010.

[3] Jinhui Hu, Ruimin Hu, zhongyuan Wang, Yang Gong, MangDuan "Kinect depth based Enhancement for low light surveillance image"978-1-4799-2341-0/13/\$31.00@2013 IEEE.

[4] Minjae Kim1, Student Member, IEEE, Dubok Park1, David K. Han2, and Hanseok Kol "A Novel Framework for Extremely Lowlight VideoEnhancement"2014 IEEE International Conference on Consumer Electronics (ICCE).

[5] Rafael C. Gonzalez, Richard E. Woods, Steven L. Eddins "Digital Image Processing using MATLAB" $2^{\text {nd }}$ edition

[6] Haralick, Robert M., and Linda G. Shapiro, Computer and Robot Vision, Volume I. Addison-Wesley, 1992.

[7] Sonka. M, Hlavac. V, Boyle. R, Image Processing, Analysis and Machine Vision, PWS Publishing, 1998 